\title{
Innovative Model of Single Target Coordinated Management in Major Constructions Based on the Timeliness of Information
}

\author{
$\mathrm{Na} \mathrm{Zhao}^{1, \mathrm{a}}$ and Shi $\mathrm{An}^{1, \mathrm{~b}^{*}}$ \\ ${ }^{1}$ School of Management, Harbin Institute of Technology,150001, China \\ azhaona@hit.edu.cn; banshi@hit.edu.cn
}

Keywords: Coordinated management; Timeliness of information; Major constructions; Game theory

\begin{abstract}
Objective: based on the game theory and agency theory, through the timeliness of information study the innovative pattern of single-target coordinated management in major projects to improve performance and income. Methods: the thesis in to optimize the coordinated management by considering a single target which is the timeliness of information between Principal and Agent in major projects, and through it to build an optimized coordinated pattern. Results: within the fixed cost of major projects, by using the coordinated optimization instead of a prescriptive management, the Principal spur the Agent to deliver and receive information actively and achieve the purpose that coordinately manage the single targeted timeliness of information and improve both sides' benefit in Pareto. Conclusion: the optimized coordinated pattern puts all related parties together in risk and benefit. This pattern will help to put the benefit of all agents i.e., the construction unit, supervising unit (company or individual) on the success of the major projects and the Principal and build a win-win relationship.
\end{abstract}

\section{Introduction}

With economy development, the number and scale of the major constructions are more and more increasing; the management in the processing is widely concerned by the government and society. The coordinated management has great value in the study of intricate system, i.e. Major constructions [1]. But most researches are on the basis of traditional view or a macroscopic view. The thesis study in a different way that a microcosmic and single view.

In the processing of major products, the timeliness of information is very important, including the instruction from the management level and the feedback from the basic level. Just like the military, Decentralized command and control settings like those found are rife with complexity and change [2]. It has close relationship to the schedule, cost and the quality [3].

With the coordination in timeliness of information, the Principal hopes the project finish in high efficiency and quality to get into operation and return the funds as soon as possible. However, in the practical construction, the information delivery is subject to many factors making it in low efficiency and coordination between the construction unit and the agent. Without the coordination in timeliness of information, in order to get a short-term benefit, the construction unit may manage roughly and use the simple way, which will cause loss. For example, because of the low-efficient in coordination, the required change by the Principal was not delivered on time, causing loss and increasing cost. therefore, the major constructions need to increase coordinate management to compensates other aspects [4-5].

In order to keep teams focused on the modular strategies, they will need to coordinate the efforts of development groups [6]. The efficiency in the timeliness of information in major constructions is essential to the cost and benefit for both sides. In the view of benefits, the coordination of all related parties is conducive to the improvement of organization relationship and the performance. In the view of coordinated optimization, a pattern of single-target coordinated management based on the timeliness of information should be constructed, which helps the coordinated management in the timeliness of information. 


\section{Problem Description}

In the processing of major constructions, normally the Principal divides the whole project into several sections for many independent agents and construct at the same time or gradually [7]. These agents do not have direct competition or cooperation. In the concurrent construction, the schedule of the whole project has close relationship to the agent who is responsible for the longest construction. So considering the cooperation between the principal and the agent as an evaluation period, that is, the degree of coordination is relatively fixed. The principal optimizes the single-targeted coordination in the timeliness of information with the key-section agents [8].

As for the principal, the basic purpose is to optimize the timeliness of information and maximize the benefit [9]. In the circumstance of coordinated optimization, though the final target is to get max benefit, the agent will try a different way that improves own ability and control objective to get coordinated benefit. among agents of major constructions, other than training courses and channels that provide learning, the incentive program is one of the executing key factor; and among informational delivering, the function of speedy search of information is becoming more and more important [10]. As long as the benefit is higher than the cost, the agent will try their best to improve own ability and control objective [11]. As shown in Fig. 1:

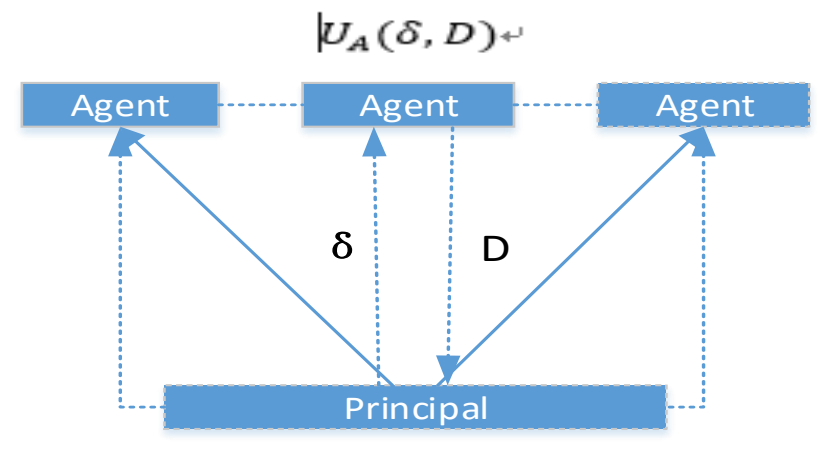

Figure 1. The collaborative management information transfer diagram

The coordination of major constructions is that the agent make a planning of the timeliness of information in coordinated project to achieve the coordinated management of the timeliness of information, according to the coordination optimization intensity given by the principal.

\section{Fundamental Assumption and Symbol Definition}

To make research easier, an assumption is made as following:

- The type of the contract made between the principal and the agent is firm lump sum plus coordinated bonus. It is signed before the starting of the construction and the funds is paid monthly; bonus is paid at the end.

- Both sides are rational

- The total cost (A) consists of direct cost (A1), indirect cost (A2) and timeliness of information coordinated cost (A3). Direct cost stands for raw material, labor cost and equipment cost. It is fixed and independent of timeliness of information coordination. Indirect cost stands for the management expense, interests, forfeit because of completion delay. Generally speaking, the longer timeliness of information, the more direct cost. This thesis assumes a positive correlation between indirect cost and timeliness of information

- The key point in the project processing is driven by resources.in limited degree, the increase of the resources can coordinate the during time to shorten the timeliness of information. The cost of major constructions is Piecewise- linearity convex function of timeliness of information. The quantity in the exponent $A(D)=N \cdot e^{-\lambda D} \quad(N$ and $\lambda$ are constants $\lambda>0$, 
$N>0$,describe the relation, the value of $N$ and $\lambda$ can be achieved by analyzing of regression, on the basis of statistic data.

If $\mathrm{D}_{0}$ stands for the normal timeliness of information; $\mathrm{A}_{0}$, the normal cost. $\mathrm{D}^{*}$, the coordinated timeliness of information; $A^{*}$, the relevant cost, the cost of coordinated timeliness of information can be shown as following:

$$
A_{3}\left(D^{*}\right)=N \cdot e^{-\lambda D^{*}}-N \cdot e^{-\lambda D_{0}}
$$

Other symbol definitions, see table 1 :

Table 1 The symbol definitions of The Innovative Model of Single-Target Coordinated Management

\begin{tabular}{|c|c|}
\hline symbol & significance \\
\hline$M$ & time limit of major projects \\
\hline$\Gamma$ & firm lump sum in the contract \\
\hline$\delta$ & $\begin{array}{l}\text { continuous decision variable. Coordination intensity for the agent given by the principal, } \\
\text { that is, the bonus awarded to the agent because of early completion of the project. } \beta>0\end{array}$ \\
\hline $\mathrm{D}_{0}$ & timeliness of information in regular construction \\
\hline$D_{\min }$ & timeliness of information in shortest period the agent \\
\hline D & $\begin{array}{l}\text { continuous decision variable. timeliness of information in construction (the actual one) the } \\
\text { agent }\end{array}$ \\
\hline$A_{0}$ & cost of the agent \\
\hline$A_{1}$ & Direct cost the agent \\
\hline$A_{2}$ & indirect cost the agent \\
\hline$a_{2}$ & indirect cost per unit time the agent \\
\hline$A_{3}$ & cost in information coordination the agent \\
\hline $\mathrm{V}_{\mathrm{p}}(\delta, \mathrm{D})$ & revenue function of the principal \\
\hline $\mathrm{V}_{\mathrm{c}}(\delta, \mathrm{D})$ & revenue function of the agent \\
\hline I & $\begin{array}{l}\text { total revenue of the principal at completion. After paying the funds at the beginning and } \\
\text { during the construction, the net cash flow displayed as benefit when the project is finished. }\end{array}$ \\
\hline $\mathrm{k}_{\mathrm{p}}$ & $\begin{array}{l}\text { expected net earnings of the principal per unit time after the official operation of the } \\
\text { project, including toll and other earnings. }\end{array}$ \\
\hline $\mathrm{K}_{\mathrm{c}}$ & opportunity benefit of the agent got from other projects \\
\hline $\mathrm{I}$ & discount rate \\
\hline
\end{tabular}

\section{Basic Model}

With the fundamental assumption and symbol definition, the linear coordinated contract between the Principal and Agent can be showed as:

$S(\delta, D)=\gamma+\delta\left(D_{0}-D\right)$

To the principal, early completion will bring operational benefit, which can be used for the bonus to part of agents and to pay the loans. But the project funds should be paid to agents, which increase loan interests. The increased and reduced interest can be canceled out partly. So the change of loan interests is negligible. The net present value function of the principal will be:

$\left.V_{p}(\delta, D)=K_{p}\left(D_{0}-D\right)\right] e^{-i D_{0}}+\left[I-\delta\left(D_{0}-D\right)\right] e^{-i D}$

$=\left(K_{\rho} e^{-i D_{0}}-\delta e^{-i D}\right)+\left(D_{0}-D\right) I \cdot e^{-i D}$

As the high-level decision maker, the responsibility of the principal is to specify the coordination intensity $\delta$ to achieve the max net present value. In another word, solve the optimization problems [12].

s.t. $\delta \geq 0$

$\left(Q_{1}\right) \max _{\delta, D} V_{p}(\delta, D)=\max _{\delta, D}\left[\left(K_{p} e^{-i D_{0}}-\delta e^{-\mathrm{i} D}\right)\left(D_{0}-D\right)+I \cdot e^{-i D}\right]$

The coordination cost in the timeliness of information of the agent:

$A_{3}(D)=N \cdot e^{-\lambda D}-N \cdot e^{-\lambda D_{0}}=N\left(e^{-\lambda D}-e^{-\lambda D_{0}}\right)$ 
As for the agent, within in the coordinated timeliness of information, they can get bonus and opportunity benefit earlier. But the coordination expense is increased. the net earnings' function of the agent is:

$$
\begin{aligned}
& V_{c}(\delta, D)=\left[\gamma-A_{1}-A_{2}-A_{3}(D)+\delta\left(D_{0}-D\right)\right]+K_{c}\left(D_{0}-D\right) \\
& =\gamma+\left(\delta+K_{c}\right)\left(D_{0}-D\right)-A_{1}-a_{2} D-N\left(e^{-\lambda D}-e^{-\lambda D_{0}}\right)
\end{aligned}
$$

As the low-level decision maker, the responsibility of the agent is to choose best timing (D) under the given coordinated intensity $(\delta)$, that is, solve the optimization problems $\left(\mathrm{Q}_{2}\right)$.

s.t. $0<D_{\min } \leq D \leq D_{0}$

$\left(Q_{2}\right) \max _{D} V_{c}(\delta, D)=\max _{D}\left[\left(\delta+K_{c}\right)\left(D_{0}-D\right)-A_{1}-a_{2} D-N\left(e^{-\lambda D}-e^{-\lambda D_{0}}\right)+\gamma\right]$

\section{Solve Models}

The agents know well of the principal's choices, because every information unit only contains one figure; and the information is delivered in order and observable. So the game is dynamic with whole information. By backward induction, the game model can be solved.

On the circumstance that the coordinated intensity $\delta$ is given, the choice $\left(Q_{2}\right)$ of the best timing is a nonlinear programming problem, $V_{\mathrm{a}}^{n}(\delta, D)=-\lambda^{2} e^{-\lambda D} N<0$, the objective function $\left(Q_{2}\right)$ is a concave function. Lagrange function is:

$$
\begin{aligned}
& M\left(D, \lambda_{1}, \lambda_{2}, \lambda_{3}\right)=\left[\left(\delta+K_{\mathrm{c}}\right)\left(D_{0}-D\right)+\gamma-A_{1}-N\left(e^{-\lambda D}-e^{-\lambda D_{0}}\right)-\mathrm{a}_{2} D\right] \\
& +\lambda_{1}\left(D_{0}-D\right)+\lambda_{2}\left(D-D_{\min }\right)+\lambda_{3} D
\end{aligned}
$$

The optimized condition is:

$$
\left\{\begin{array} { l } 
{ D \geq 0 , \frac { \partial M } { \partial D } \leq 0 , D \frac { \partial M } { \partial D } = 0 } \\
{ \lambda _ { 1 } \geq 0 , \frac { \partial M } { \partial \lambda _ { 1 } } \geq 0 , \lambda _ { 1 } \frac { \partial M } { \partial \lambda _ { 1 } } = 0 } \\
{ \lambda _ { 2 } \geq 0 , \frac { \partial M } { \partial \lambda _ { 2 } } \geq 0 , \lambda _ { 2 } \frac { \partial M } { \partial \lambda _ { 2 } } = 0 } \\
{ \lambda _ { 3 } \geq 0 , \frac { \partial M } { \partial \lambda _ { 3 } } \geq 0 , \lambda _ { 3 } \frac { \partial M } { \partial \lambda _ { 3 } } = 0 }
\end{array} \stackrel { D > 0 } { \longrightarrow } \left\{\begin{array}{c}
\frac{\partial M}{\partial D}=N \lambda e^{-\lambda D}-\delta-a_{2}-K_{c}-\lambda_{1}+\lambda_{2}+\lambda_{3}=0 \\
\lambda_{1} \frac{\partial M}{\partial \lambda_{1}}=\lambda_{1}\left(D_{0}-D\right)=0 \\
\lambda_{2} \frac{\partial M}{\partial \lambda_{2}}=\lambda_{2}\left(D-D_{\text {min }}\right)=0 \\
\lambda_{3} \frac{\partial M}{\partial \lambda_{3}}=\lambda_{3} D=0 \stackrel{D>0}{\longrightarrow} \lambda_{3}=0
\end{array}\right.\right.
$$

$\lambda_{1} 、 \lambda_{2} 、 \lambda_{3}$ are Lagrange coefficients.

Lagrange coefficients $\lambda_{1} \geq 0, \lambda_{2} \geq 0$, there are four combinations. But there is an implied condition that $D_{0}>D_{\min }$, so $\lambda_{1}, \lambda_{2}$ cannot be positive at the same time, otherwise, $D_{0}=D_{\min }$.

On this circumstance, there are three situations as following:

(1) : $\lambda_{1}>0, \lambda_{2}=0$, the condition is as following:

$$
\left\{\begin{array}{c}
\frac{\partial M}{\partial D}=N \lambda e^{-\lambda D}-\delta-a_{2}-K_{c}-\lambda_{1}+\lambda_{2}+\lambda_{3}=0 \\
\frac{\partial M}{\partial \lambda_{1}}=D_{0}-D=0 \\
\frac{\partial M}{\partial \lambda_{2}}=D-D_{\text {min }}=0 \\
\lambda_{3}=0
\end{array}\right.
$$


And then, get $D=D_{0}, \lambda_{1}=N \lambda e^{-\lambda D_{0}}-\delta-a_{2}-K_{c}, \lambda_{2}=0, \lambda_{3}=0$, and $\delta<N \lambda e^{-\lambda D_{0}}-\mathrm{a}_{2}-K_{c}$. On this condition that $\delta<N \lambda e^{-\lambda D_{0}}-\mathrm{a}_{2}-K_{c}$, to the agent will not chose to coordinate timeliness of information. As for the principal, the best choice is $\delta=0$. The noninferior solution of (Q) is $(\delta, D)=\left(0, D_{0}\right)$.The earnings of the principal is $V_{P}=e^{-\mathrm{iD}_{0}} \cdot I$, the earnings of the agent is $V_{a}=-A_{1}+\gamma-a_{2} D_{0}$.

(2) $\lambda_{1}=0, \lambda_{2}>0$, the condition is as following:

$$
\left\{\begin{array}{c}
\frac{\partial M}{\partial D}=N \lambda e^{-\lambda D}-\delta-a_{2}-K_{c}-\lambda_{1}+\lambda_{2}+\lambda_{3}=0 \\
\frac{\partial M}{\partial \lambda_{1}}=D_{0}-D>0 \\
\frac{\partial M}{\partial \lambda_{2}}=D-D_{\text {min }}=0 \\
\lambda_{3}=0
\end{array}\right.
$$

Get $D=D_{\min }, \lambda_{1}=0, \lambda_{2}=-N \lambda e^{-\lambda D_{\min }}+\delta+a_{2}+K_{c}, \lambda_{3}=0$. On this condition that $\delta>N \lambda e^{-\lambda D_{\min }}-\mathrm{a}_{2}-K_{c}$, the agent will choose to coordinate production period with maximization.

As for the project company, the best choice is $\delta=N \lambda e^{-\lambda D_{\min }}-a_{2}-K_{c}+\omega$ ( $\omega$ is an infinitesimal positive number). The noninferior solution of $\mathrm{Q}$ is $(\delta, D)=\left(N \lambda e^{-\lambda D_{\min }}-a_{2}-K_{c}+\omega, D_{\min }\right)$. The earnings of the principal is $V_{p}=I \cdot e^{-i D_{\min }}+\left(D_{0}-D_{\min }\right)\left[K_{p} e^{-\mathrm{i} D_{0}}\left(N \lambda e^{-\lambda D_{\min }}-a_{2}-K_{c}+\omega\right) e^{-\mathrm{i} D_{\min }}\right]$, the earnings of the agent is $V_{c}=\gamma+\left(D_{0}-D_{\min }\right)\left(N \lambda e^{-\lambda D_{\min }}-\mathrm{a}_{2}+\omega\right)-N\left(e^{-\lambda D_{\min }}-e^{-\lambda D_{0}}\right)-A_{1}-\mathrm{a}_{2} D_{\min }$.

(3) $\lambda_{1}=0, \lambda_{2}=0$, the condition is as following:

$$
\left\{\begin{array}{c}
\frac{\partial M}{\partial D}=N \lambda e^{-\lambda D}-\delta-a_{2}-K_{c}-\lambda_{1}+\lambda_{2}+\lambda_{3}=0 \\
\frac{\partial M}{\partial \lambda_{1}}=D_{0}-D=0 \\
\frac{\partial M}{\partial \lambda_{2}}=D-D_{\text {min }}>0 \\
\lambda_{3}=0
\end{array}\right.
$$

acquire: $D_{\min }<D=-\frac{1}{\lambda} \ln \frac{\delta+\mathrm{a}_{2}+K_{c}}{N \lambda}<D_{0}, \lambda_{1}=0, \lambda_{2}=0, \lambda_{3}=0$.

Reach: $N \lambda e^{-\lambda D}-a_{2}-K_{c}<\delta<N \lambda e^{-\lambda D_{\min }}-\mathrm{a}_{2}-K_{c}$

When the project company decide incentive intensity $\delta$, they forecast that the agent will chose the best time limit by $D=-\frac{1}{\lambda} \ln \frac{\delta+a_{2}+K_{c}}{N \lambda}$. The max target of present value of earnings (4) can transform the two-layer optimization problem into single-layer one $(\mathrm{Q})$ :

$$
\begin{aligned}
& (Q) \max _{\delta} V_{p}(\delta)=\max _{\delta}\left[\left(D_{0}+\frac{1}{\lambda} \ln \frac{\delta+a_{2}+K_{c}}{N \lambda}\right)\left(K_{p} e^{-\mathrm{i} D_{0}}-\delta e^{\frac{i}{\lambda} \ln \frac{\delta+a_{2}+K_{c}}{N \lambda}}\right)+I \cdot e^{\frac{i}{\lambda} \ln \frac{\delta+a_{2}+K_{c}}{N \lambda}}\right] \\
& \text { s.t.x }<\delta<y
\end{aligned}
$$

In figure (17): $\mathrm{x}=N \lambda e^{-\lambda D_{0}}-a_{2}-K_{c}, \mathrm{y}=N \lambda e^{-\lambda D_{\min }}-a_{2}-K_{c}$. 


\section{Genetic Algorithm of Coordinated Management}

However, it's a typical nonlinear programming problem, both objective function (16) and constraint equation (17) are nonlinear. It is difficult to solve this problem by any traditional optimization method. Genetic algorithm is a probability algorithm with global optimization by simulating the inheritance and evolution process in biology.

This algorithm is to repeatedly filtrate, cross the encoded string groups to generate more adapted group to new environment. By iterative computations, a best or quasi-optimal solution will be achieved and no restriction on the searching of the objective function. Thus, Genetic algorithm is as following:

- Initialization: set evolution algebra calculator $\mathrm{g} \leftarrow 0$ : set the maximum evolution algebra W generate initialized group pop $(\mathrm{g})$ to $\mathrm{h}$ kinds of individual with random. The binary coding length of each one is 1 , a solution can be achieved

- Ideal adaptation evaluation: Define the objective function in (16) as an individual fitness function and decode the individual position in the $\operatorname{pop}(\mathrm{g})$ to get the truth of $\theta$. then calculate the fitness degree $f \mathrm{n}, \mathrm{n}=1.2 \ldots, \mathrm{h}$ :

- Selecting operation: $P_{\mathrm{n}}=f_{n} / \sum_{n=1}^{h} f_{n}$ chose h units randomly from pop(g), and form a new group newpop $(\mathrm{g})=\{\operatorname{pop}(g) \mid n=1,2, \cdots, h\}$, new $\operatorname{pop}(\mathrm{g})$ may choose some factors from $\operatorname{pop}(\mathrm{g})$

- Interlace peration: stochastically play the crossover probability $\mathrm{Pz}$ (normally $0.6 \leq \mathrm{Pz} \leq 1.0$ ) on newpop(g) to get $\operatorname{crosspop}(\mathrm{g})$ with $\mathrm{h}$ individuals.

- Mutation operation: stochastically play the smaller mutation probability $\mathrm{Pz}$ (normally $0.001 \leq \mathrm{Pz} \leq 0.01)$ on cross $\operatorname{pop}(\mathrm{g})$ to get mutpop $(\mathrm{g})$.

- End condition: if $\mathrm{g} \leq \mathrm{W}, \mathrm{g} \leftarrow 1+\mathrm{g}$, go back to step 2; if $\mathrm{g}>\mathrm{W}$, the optimal solution is the individual with the highest fitness degree. the calculation is finished.

Therefore, the total revenue of the principal and the expected net earnings in operation per unit time is positive to the optimal coordination intensity at completion of the project. In another word, the optimal coordination intensity is increasing with the growth of "I". But the increasing range will continuously weaken to the upper limit, the agent will coordinate the timeliness of information within the reasonable range to increase benefit of both sides. The indirect cost per unit time A2 and opportunity benefit of the agent $\mathrm{KC}$ is in negative correlation with optimal coordination intensity. In another word, the bigger $\mathrm{A} 2$ and $\mathrm{KC}$, the more active of the agent to coordinate project time. Even if the coordination intensity is reduced, the agent will continuously coordinate the timeliness of information within the reasonable range to lower the indirect cost and gain more opportunity benefit.

\section{Conclusion}

Based on the game theory of the specific major constructions, the thesis combines the objectives of Principal and Agent together by the coordination optimization pattern. The paper structured and analyzed the innovative pattern of single-target coordinated management in major constructions. The result indicates that within the fixed cost of major constructions, by using the coordinated optimization instead of a prescriptive management, the Principal spur the Agent to actively coordinate the timeliness of information, with the purpose of coordination management and the improvement of both sides' benefit in Pareto. The implement of the coordination optimization contract combines all related parties to share risk and benefit. This Innovative model will help combine the benefit of all agents with the success of the major constructions and the Principal to build a win-win relationship. 


\section{Acknowledgement}

This work was supported by the National Natural Science China (Grant No. 71390522), China Postdoctoral Science Foundation and Hei Long Jiang Postdoctoral Foundation of China.

\section{References}

[1] Potkány, M. (2015). Coordinated management model of support business processes through the facility management. Procedia Economics \& Finance, 23, 396-401.

[2] Gorman, Jamie C.; Cooke, Nancy J. \& Winner, Jennifer L. (2006). Measuring team situation awareness in decentralized command and control environments. Ergonomics, 49(12-13), 1312-1325.

[3] Lavikka, RH; Smeds, R \& Jaatinen, M (2015). Coordinating collaboration in contractually different complex construction projects. Supply Chain Management-An International Journal, 20(2), 205-217.

[4] Gerwin, D. \& Barrowman, NJ. (2002). An evaluation of research on Integrated Product Development. Management Science, 48(7), 938-953.

[5] Marle, Franck; Vidal, Ludovic-Alexandre. (2014). Forming Risk Clusters in Projects to Improve Coordination between Risk Owners. Journal of Management in Engineering, 30(4), 06014001.

[6] Baldwin, CY. \& Clark, KB. (1997). Managing in an age of modularity. Harvard Business Review. 75(5), 84-\&.

[7] Madhusudan, T. (2005). An agent-based approach for coordinating product design workflows. Computers in Industry. 56(3), 235-259.

[8] Tserng, HP \& Lin, YC. (2004). Developing an activity-based knowledge management system for contractors. Automation in Construction, 13(6), 781-802.

[9] Hjaila, K., Zamarripa, M., Shokry, A., \& Espuña, A. (2014). Application of pricing policies for coordinated management of supply chains. Computer Aided Chemical Engineering, 33, 475-480.

[10] Yeh, Ying-Jung; Lai, Sun-Quae \& Ho, Chin-Tsang (2006). Knowledge management enablers: a case study. Industrial Management \& Data Systems, 106(5-6), 793-810.

[11]Kandrashina, E. A., \& Ashmarina, S. I. (2015). Economic interests as the basis for coordinated management of organizational changes. Mediterranean Journal of Social Sciences. 6(6), S3.

[12]Petrie, Cj; Webster, Ta \& Cutkosky, Mr. (1995). Using Pareto Optimality to Coordinate Distributed Agents. Ai Edam-Artificial Intelligence for Engineering Design Analysis and Manufacturing, 9(4), 269-281. 